Article

\title{
Construction of a Decision Support System Based on GP Services, Using a Warning-Judgment Module as an Example
}

\author{
Xuejia Sang ${ }^{1}$, Linfu Xue ${ }^{1, *}$, Xiaopeng Leng ${ }^{2,4}$, Jiwen Liu ${ }^{1}$ and Liang Zhan ${ }^{3,4}$ \\ 1 College of Earth Science, Jilin University, Changchun 130061, China; sangxj15@mails.jlu.edu.cn (X.S.); \\ jwliu2213@mails.jlu.edu.cn (J.L.) \\ 2 Key Laboratory of Mountain Hazards and Earth Surface Process, Chinese Academy of Science, \\ Chengdu 610041, China; lengxiaopeng@cdut.cn \\ 3 Xinjiang Uyghur Autonomous Region Academy of Surveying and Mapping, No. 231 Sandaowan East Road, \\ Urumqi 830002, China; jishu@casm.ac.cn \\ 4 College of Information Sciences and Technology, Chengdu University of Technology, Chengdu 610059, China \\ * Correspondence: xuelf@jlu.edu.cn; Tel.: +86-176-498-119-87
}

Academic Editors: Milan Konecny and Wolfgang Kainz

Received: 30 March 2017; Accepted: 1 June 2017; Published: 5 June 2017

\begin{abstract}
Decision-making departments need more detailed and timely data in order to meet the needs of emergency response. Sichuan province is an area that frequently suffers natural disasters, and many disasters are caused by rainfall. This study establishes a decision support system (DSS) based on geoprocessing (GP) services, which can locate the region that overran the rainfall threshold and provide the population or property analysis, query, map plot, and path analysis functions. Most of the functions of the system are developed on the basis of geoprocessing services. This paper uses the warning-judgment module as an example to introduce the structure and function of the DSS system. The system satisfies the demands of real-time data acquisition, calculation, analysis, and presentation.
\end{abstract}

Keywords: GIS; geoprocessing; DSS; ArcGIS Server; SDK; Flex; geoinformation

\section{Introduction}

The World Meteorological Organization (WMO) estimates that the disaster reduction rate owing to decision support systems (DSS) accounts for approximately $10-15 \%$ of the total benefits of global disaster reduction 1 . Therefore, the construction of DSS is a matter of great urgency. The secondary development phase of most emergency command systems based on geographic information systems (GIS) has been established, so the DSS itself needs to display, insert, and process a large amount of spatial data. With the rapid development of Web technology and GIS, GIS technology based on the Web plays an increasingly important role in DSS. Independent of clients, a Web-based display terminal has a simple interactive interface and a simple deployment of B/S (browser/server) architecture. Except for the requirement of server performance, its advantages include low hardware cost, separately arranged modules and data, and easy upgrading and data sharing.

In recent years, there has been a large amount of research into Web- and GIS-based decision support systems. Engel built a Web-DSS system based on Oracle and MapServer GIS Web with an L-THIA model [1]. The system provides the functions of map display, data statistics, and so on, which provides technical support for the user's hydrological decision. By computer software and hardware conditions, the computing power is poor and lacks pertinence [2]. Salah used Apache to build a water pollution management decision system (WaterDSS), building a calculation module based on PHP, which is a popular general-purpose scripting language that is especially suited to web development, 
to communicate with the Ajax-based foreground program. This system achieves the inclusion of water resources data and map display functionality [3]. Kulkarni established a hydrological model for the Koparkhairane and Kalamboli watersheds, and created a browser-based city waterlogging assessment and decision system named the integrated flood assessment model (IFAM) [4], the advantages of which include high usability and expansibility, though its simulation scope covers too small an area (5 km). Chen [5] constructed a disaster management platform based on an ArcGIS server and Twitter which can identify different disasters through query and provide a hot map preview. Based on the big data of Twitter, the system has a certain functionality for emergency disaster management, but it still needs manual updating, and its lack of a keywords filter consequently leads it to misjudge the subjective assessments of Twitter users. With the application of the ArcGIS network analysis function, Zhang et al. [6] created a Jingjiang flood decision support system though which people who live in a flood-relief area can plan for transfer routes and estimated times of arrival, and the system can also provide real-time traffic guidance. $\mathrm{Wu}$ [7] analogously designed a system named the weather service product generation system (WSPGS); through geoprocessing services, it can design workflow according to the demands of the users. Although WSPGS is used primarily to output maps on the basis of meteorological data, it can be seen as a prototype of DSS. In order to ensure its timeliness, the ArcGIS server is used to deploy geoprocessing services. This system has the huge advantage of receiving remote sensing data through the Internet [8-10], and works using geoprocessing services, defining its process online with selected attributes of geoprocessing services, and ensuring high efficiency and stability.

In recent years, there has been much research on Web-based DSS technology, but most of the systems that have been developed have the same problem: their functionality is simple and their expansibility is poor [11,12]. About 10 years ago, most scholars published their models by rewriting PHP or JS [13]. In recent years, many GIS companies have released their server products to reduce the difficulty of building GIS systems, while also enhancing the stability of those systems. However, research into building GIS-based DSS systems is rare [14]. The main difficulty lies in improving the efficiency and stability of the system logic [15-17]. After referring to previous work, we designed a DSS based on geoprocessing services. The method of contrasting current data to historical maximum rainfall data is applied here to locate rainstorm areas. Therefore, our system can provide early warning timeliness, determining the warning area in time and ensuring the stability and timeliness of the system.

The strong ability to manage spatial data is expanded according to its rainfall warning model, and some basic tools about processing geographic information are designed [18]; thus, the rainfall warning model of DSS has been established. By virtue of its feature of simplicity, easy customization, and deployment, we created a set of decision support systems which can meet the requirements of data integration, task scheduling, and high stability. This paper first introduces the real-time rainfall warning module of the emergency command system in Sichuan Province, then introduces the algorithm foundation, deployment, and application. This example has been run in the emergency command system Intranet of Sichuan Province, and the operation effect is good. Research shows that, thanks to the high stability and scalability of ArcGIS Server, with the use of the assembled geoprocessing (GP) services in the background, you can achieve the needs of most DSS functional requirements.

\section{Research Area and System Introduction}

Sichuan Province (Figure 1) has a total land area of 485 thousand $\mathrm{km}^{2}$, which renders it the fifth broadest land area among 31 provinces. Located in western China, Sichuan has extraordinarily abundant landscape types, of which the main ones contain not only plains and mountains but also hilly and plateau terrain. Sichuan Province is located in a subtropical region, and there are three terrains: the Sichuan Basin, Southwest Sichuan Mountain, and the mountain climate zone of northwest Sichuan. The climate of Sichuan is complex and varied. Drainage in Sichuan Province is distributed widely; the number of rivers whose drainage area covers more than $100 \mathrm{~km}^{2}$ is more than 1000 . Because of its 
location in the medium of the North-South Seismic Belt, seismic activity frequency in Sichuan Province is relatively high. Sichuan Province has suffered many kinds of disasters in history, including lightning, flood burst, earthquake, hail, and forest fires. Causing great loss to the national economy, these disasters are big threats to life and property to various extents, and also test the local government's emergency management ability. There were seven large-scale heavy rainfalls in 2007, including a hundred-year storm in one local area in July. In 2010, Sichuan Province repeatedly suffered heavy rains and floods, especially the ' 7.15 ', '7.22' rainstorm and flood disasters, and ' 8.13 ', the most serious flash flood and debris flow. In 2011, north and east Sichuan continuously suffered rainstorm and flood disasters which caused 119 people to die and 50 people to go missing; 15 heavy rainfalls and 5 regional rainstorms hit Sichuan Province in the flood season of 2012, and the average number of rainy days was 95. As a result, 31,540,000 people were affected, including 163 people dead. From 29 June to 17 July in 2013, the province suffered four strong rainfalls and 469 rainstorms.

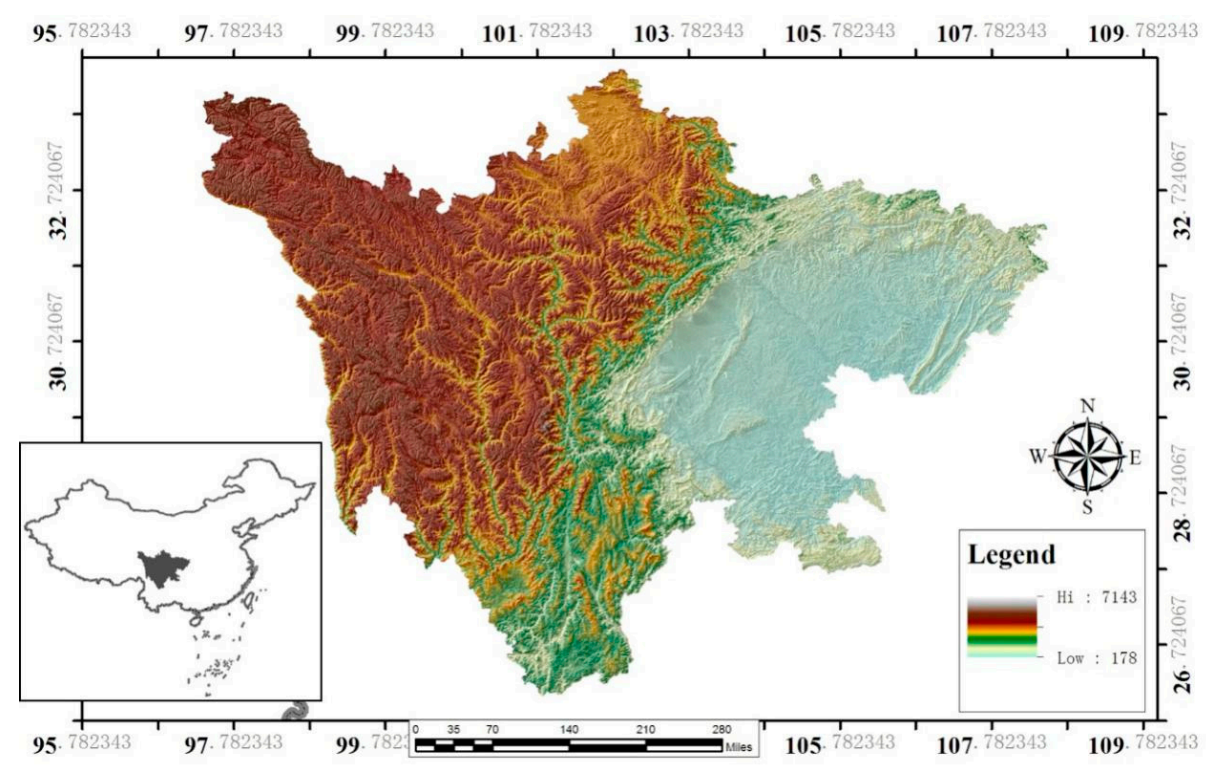

Figure 1. Topographic map of Sichuan.

In 2007, the law on emergency response in the People's Republic of China was officially promulgated and implemented, laying a legal foundation for developing an emergency industry. This emergency industry has been accepted by the central government, and has been highly valued. In response to the characteristics of natural disasters in Sichuan Province, in 2014, the Sichuan provincial geological disaster emergency headquarters was set up. At the same time, the Sichuan provincial geological disaster emergency management GIS system was established online.

Developed by Adobe Flex, the Sichuan DSS client communicates with geoprocessing by ESRI for Flex API. ESRI is the company that built ArcGIS, the world's most powerful mapping and spatial analytics software. The principle of geoprocessing has been implemented by ArcGIS products from ESRI Corporation, which also provides Model Builder and Workflow Manager tools to support normal geographical tasks. The fundamental idea of geoprocessing aims at automatically finishing GIS tasks by using multiple-step procedures known as workflows. Automation is realized by a series of tools and a mechanism of combining these tools by a sequence of operations by using models and even scripts. Each geoprocessing tool performs a small but essential operation on geographical data, such as interpolating the data set from a point data set, clipping a territory by using another polygon shape, or creating a buffer zone around features. Geoprocessing normally contains an advanced looping and branching model to support the simulation of reality.

The system utilizes a layered architecture, and the data registration center (DRC) accomplishes centralized management of all data, including Internet data, spatial data, and thematic data. The server 
layer is mainly composed of a geoprocessing services executor and model builder. The client page launches a task request to the invoker, then the invoker calls different data according to different task instructions for different geoprocessing processes, and provides feedback on task progress to Task Monitor in real time. At the end of the task, the result or index will be sent back to the foreground in JSON format. Different function modules in DSS call for different geoprocessing services and different data. Benefiting from geoprocessing services deployment and expansion, and data management by the DRC, the function and application of DSS has been greatly improved. Figure 2 shows the architecture diagram of DSS.

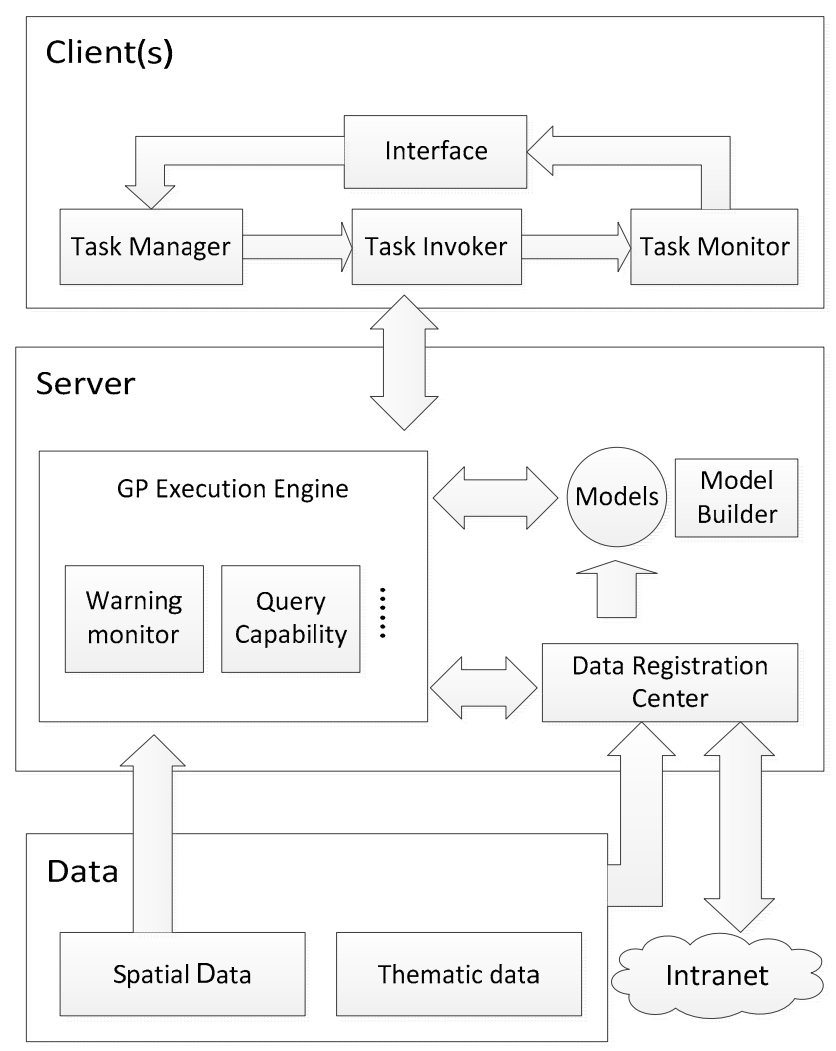

Figure 2. The architecture diagram of the decision support system (DSS). GP: geoprocessing.

\section{Method}

\subsection{GP Service}

Geoprocessing (GP) services contain geoprocessing tasks, and a task takes simple data captured in a web application, processes it, and returns meaningful and useful output in the form of features, maps, reports, and files. The possibilities for these services are infinite [19,20]. A geoprocessing service contains one or more geoprocessing tasks. A geoprocessing task is a geoprocessing tool running on a server, and its execution and outputs are managed by the server. When sharing a geoprocessing result as a geoprocessing service, a corresponding geoprocessing task is created from the tool that created the result. "Task" is a term that web-based APIs use to describe routines that do work on a server and return results. Geoprocessing services and the tasks within are used by web clients. The primary clients are web applications developed with JavaScript, SilverLight, or Flex. These latter clients use standard Internet browsers or custom-built applications to display and interrogate geography.

The DSS system mainly consists of a rainfall processing module, a judging and warning module, and a query module. All of them are built based on GP services. The task of the rainfall processing module is processing the rainfall data returning from the Intranet and storing it to the server for the 
warning module, after geocoding the rainfall data to geo-information data. The judging and warning module determines whether to provide early warning by comparing real-time rainfall to historical threshold rainfall. The query module receives requests from the client's task invoker, then feeds back other information about the location in the request by using spatial location information as query parameters. Methods used here mainly include geocoding, interpolation, judgment, and query.

\subsection{Geocoding}

Rainfall data obtained from the Intranet has no geographical location, but the model analysis procession needs different attributes along with location. The system obtains each rainfall station's location and ID in advance for storing in the data register center, because rainfall station positions are fixed. When receiving real-time rainfall data, the system will create a new dataset in the form of Points and add ID, location attributes, and other fields, and then send them to GP execution and transfer them to raster data by interpolated formatting.

\subsection{Interpolation}

All attribute values on a geographic surface are related to each other, but closer values are more strongly related than more distant ones [21] Rainfall stations in Sichuan cannot completely cover all the ground, so in order to ensure the scope of the forecast, the system uses an interpolation method to fill the missing data. There are many popular interpolation methods, mainly including the IDW (Inverse Distance Weighted) method, Kriging, and Cokriging, etc. It is generally acknowledged that IDW has low precision with a narrow scope of application; the Kriging method set weight is based on the point value around it, so its spatial correlation is better than IDW. Based on Kriging, the Cokriging method uses multiple correlated values of weight, and the precision of its result is higher. It is not the best choice to process data by the Cokriging method using only rainfall data, so we only compare the IDW and Kriging interpolation results. Because the data difference for rainfall per hour is much too small, we chose the full-time rainfall of the whole Sichuan province in order to increase the contrast. Figure 3 shows the interpolation results.

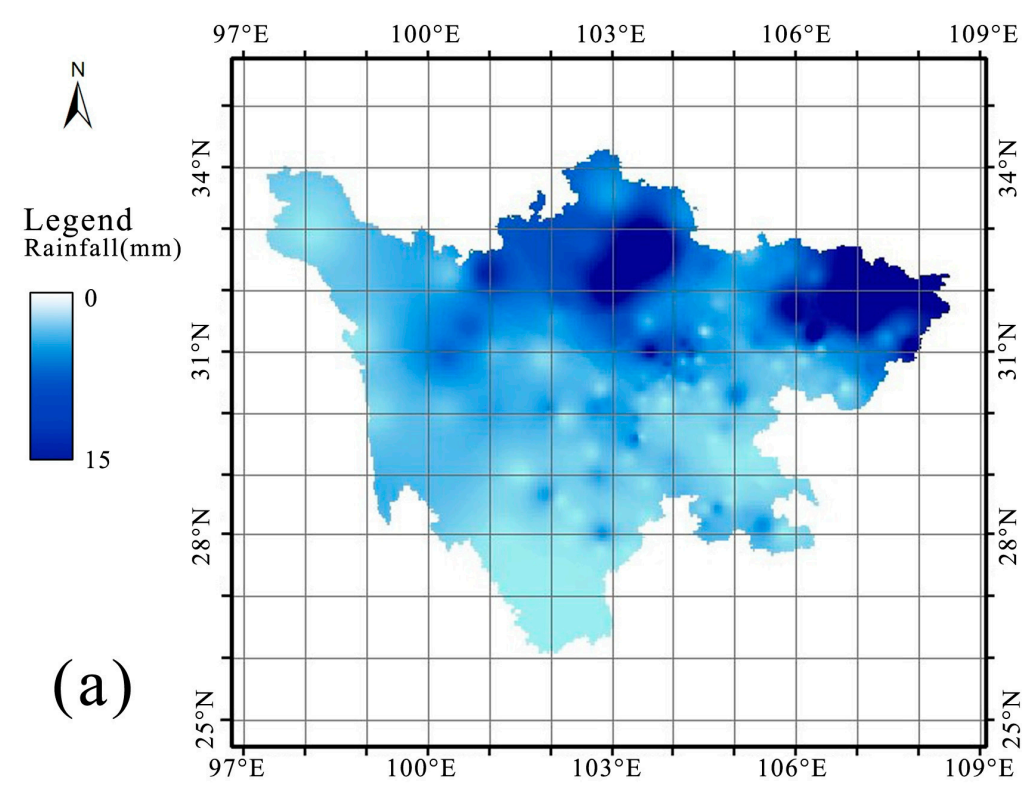

Figure 3. Cont. 


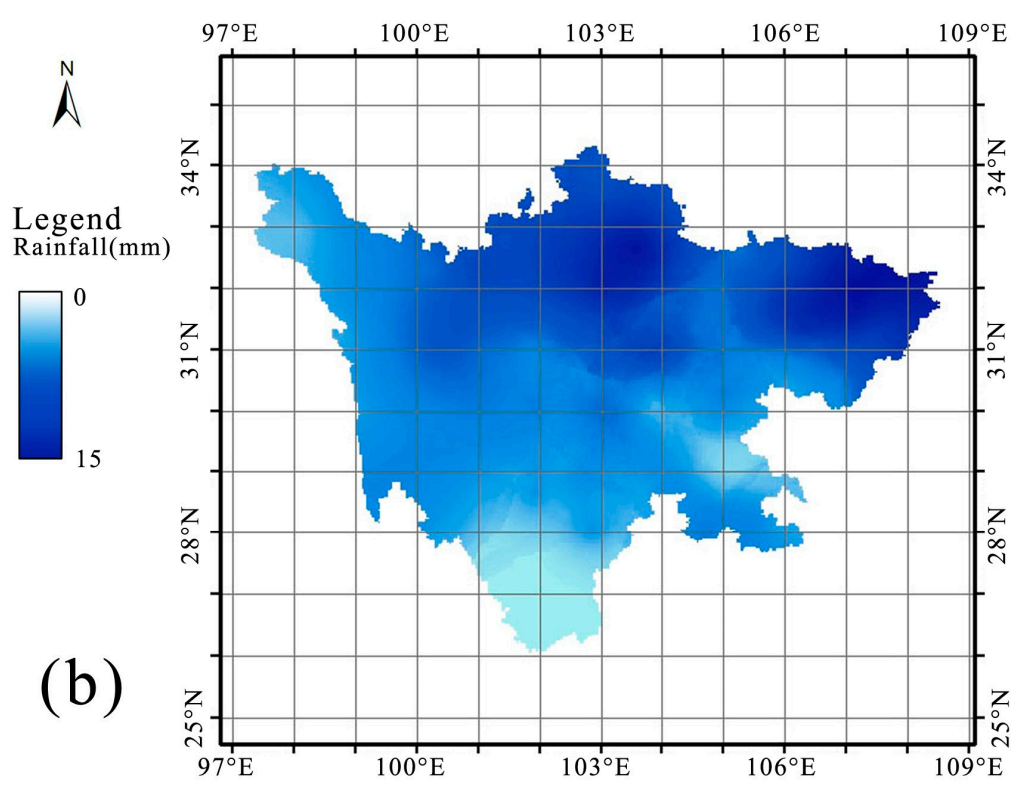

Figure 3. Comparison between the IDW (Inverse Distance Weighted) and Kriging methods: (a) IDW interpolation of rainfall (19 April, 2015); (b) Kriging interpolation of rainfall (19 April, 2015).

Compared with the Kriging rainfall results, IDW results (Figure 3a) show that there are many data outliers, especially in the center of Sichuan, whereas the Kriging interpolation results (Figure 3b) appear more naturally. Therefore, the model here uses the Kriging method to interpolate rainfall data to ensure the accuracy and reliability of interpolation results.

\subsection{Judgment}

A large number of water conservancy facilities built based on historical river flood levels were used for reference when determining disaster response levels, and rainfall is the main cause of flood disaster. Therefore, average annual maximum rainfall can be used to assess the capacity of water conservancy facilities against flood in the region. The basic data for the early warning-judgment decision comes from the average precipitation per hour chart (Figure 4a) and data from the past $24 \mathrm{~h}$ (Figure 4b) in Sichuan Province, which is taken from the "Sichuan Small and Middle Watershed Rainstorm Flood Calculation Manual" (Sichuan Provincial Water Resources Department).

The rainfall warning decision uses the matrix calculation method. The 1-h rainfall measurement and cumulative rainfall over $24 \mathrm{~h}$ measurement are respectively subtracted from the judgment base, and the remaining values show the amount and location of the warning data.

$$
\mathrm{E}=\mathrm{R}-\mathrm{R}_{0}=\left[\begin{array}{ccc}
r_{i j}-r_{0 i j} & \cdots & r_{i}-r_{0 i} \\
\vdots & \cdots & \vdots \\
r_{j}-r_{0 j} & \cdots & r_{i j}-r_{0 i j}
\end{array}\right]
$$

Equation (1) shows the original judgment data, real-time rainfall in spatial position $(i, j)$. After completion of the calculation, the warning matrix $\mathrm{E}$ will be sent to the client as a result of the judgment [22,23]. 


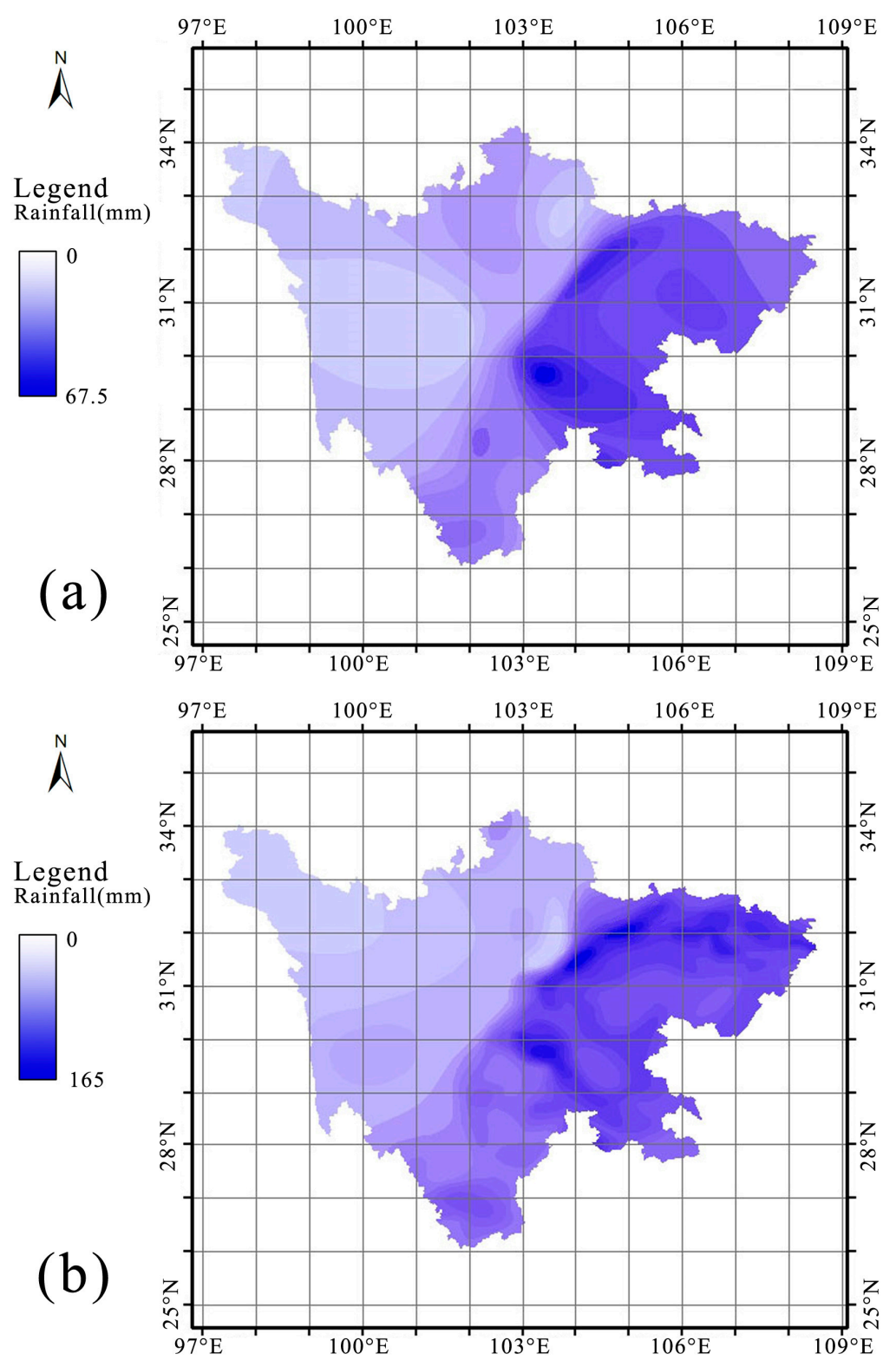

Figure 4. Rainfall data quoted from the "Sichuan small and middle watershed rainstorm flood calculation manual": (a) 1-h maximum rainfall isosurface; (b) 24-h maximum rainfall isosurface.

\section{Design and Implementation}

The core function of the system is the geoprocessing (GP) model that is released by the actuator, including the interpolation of geoprocessing services, query GP services, and early warning-judgment geoprocessing services. The interpolation of GP services (Figure 5) used a Kriging interpolation model established by the ArcGIS model builder. After completing the operations of inputting parameters, integrating the grid, masking the region, and transforming the raster to feature, we output data in the form of a polygon and overlap layers in the client. The structure of the interpolated GP model is shown in Figure 6.

The query GP model (Figure 6) can query the attributes of all layers and location data, through which we can query rainfall, population, economic losses, and some others. The location parameter in Figure 6 is the most important parameter to get the result. When a query is initiated by the proscenium, the location parameter can be sent to the task manager and formatted as a dataset in MapPoint format, 
then sent to the GP actuator. The GP actuator can acquire different values by choosing an index from different layers for different requests.

The operation of passing parameters is restricted in the Web client, and the transmission of raster data cannot meet the requirements of timeliness. Therefore, in the DSS process, we send a formative dataset in JSON format. For ArcGIS REST API can only use the GET method, which utilizes a URL to send parameters and submit data, though the size of the data parameters is highly restricted by the browser and operating system. So, we override the submitted part of the HTTP of API using the method of POST (Algorithm 1; Figure 7). Warning-judgment geoprocessing services compare the 1-h and 24-h real-time rainfall raster data after interpolation with the grid data of average annual maximum rainfall and extract the region where the rainfall is greater than average, then transform the data into a vector polygon file and send the data back to the client. The process of the warning-judgment GP model is shown in Figure 8.
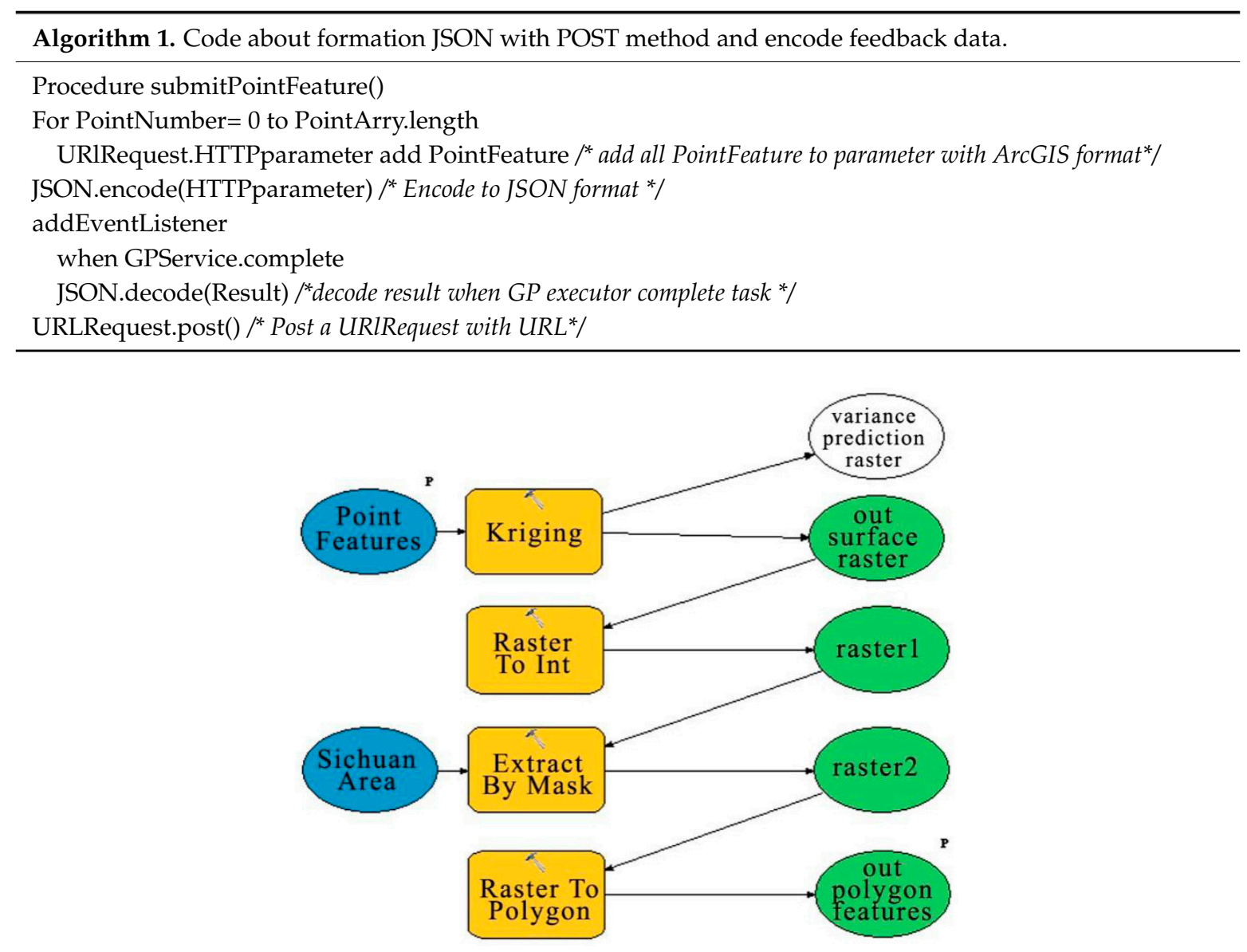

Figure 5. Interpolation GP model.

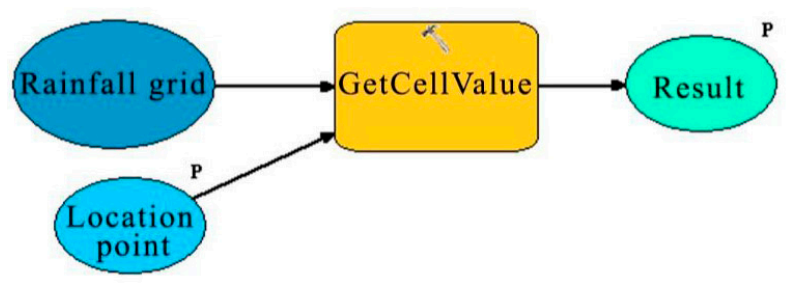

Figure 6. Query GP model. 


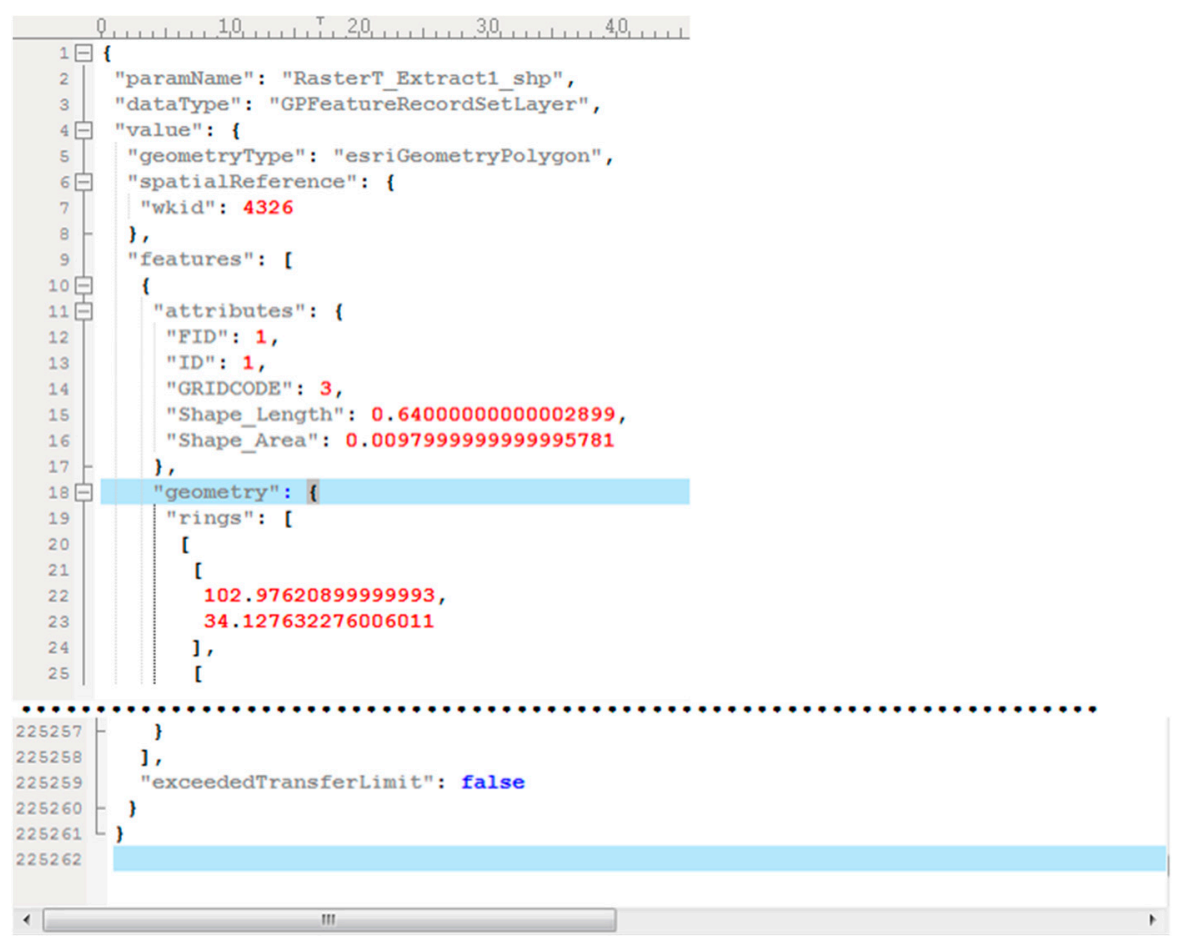

Figure 7. A feedback data case with 225,262 rows and about 8.3 MB.

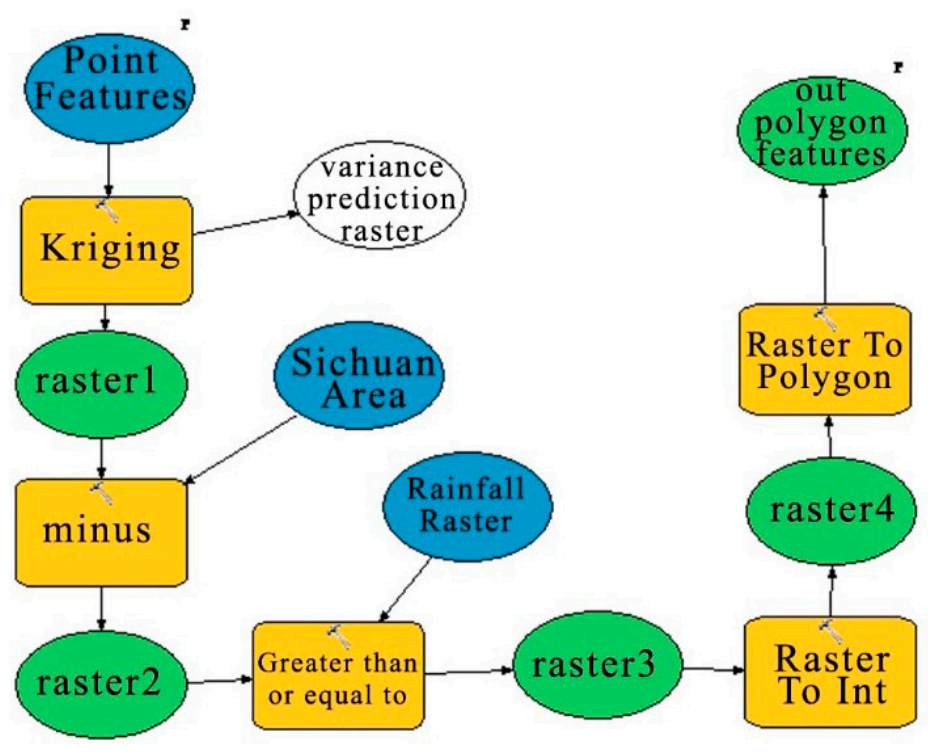

Figure 8. Warning-judgment GP model.

The DSS proscenium is based on Flex and ArcGIS REST API, and adjusts the alpha value to $40-50 \%$ to optimize the coverage effect when drawing a graphic layer. By releasing the geoprocessing services to the ArcGIS Server and completing the backstage deployment of the warning-judgment module, users can receive rainfall warning information at the map canvas in regular time.

Two figures show how rainfall is shown in the rainfall warning module of the DSS: rainfall data from low to high, and the overburden layer, from shallow to deep (Figure 9). Rainfall "tips" can pop up when clicking the mouse on the layer. If the rainfall is enough to trigger the alarm, the warning area will be in red (Figure 10). In this way, the rainfall warning-judgment process based on geoprocessing services is completed. Users only need to submit a request in the foreground, and the GP invoker will 
define the parameters of the geoprocessor, then the GP actuator will call on related data calculations, return results, and complete the task. By means of this method, DSS not only makes operation simple, but also separates presentation from calculation.

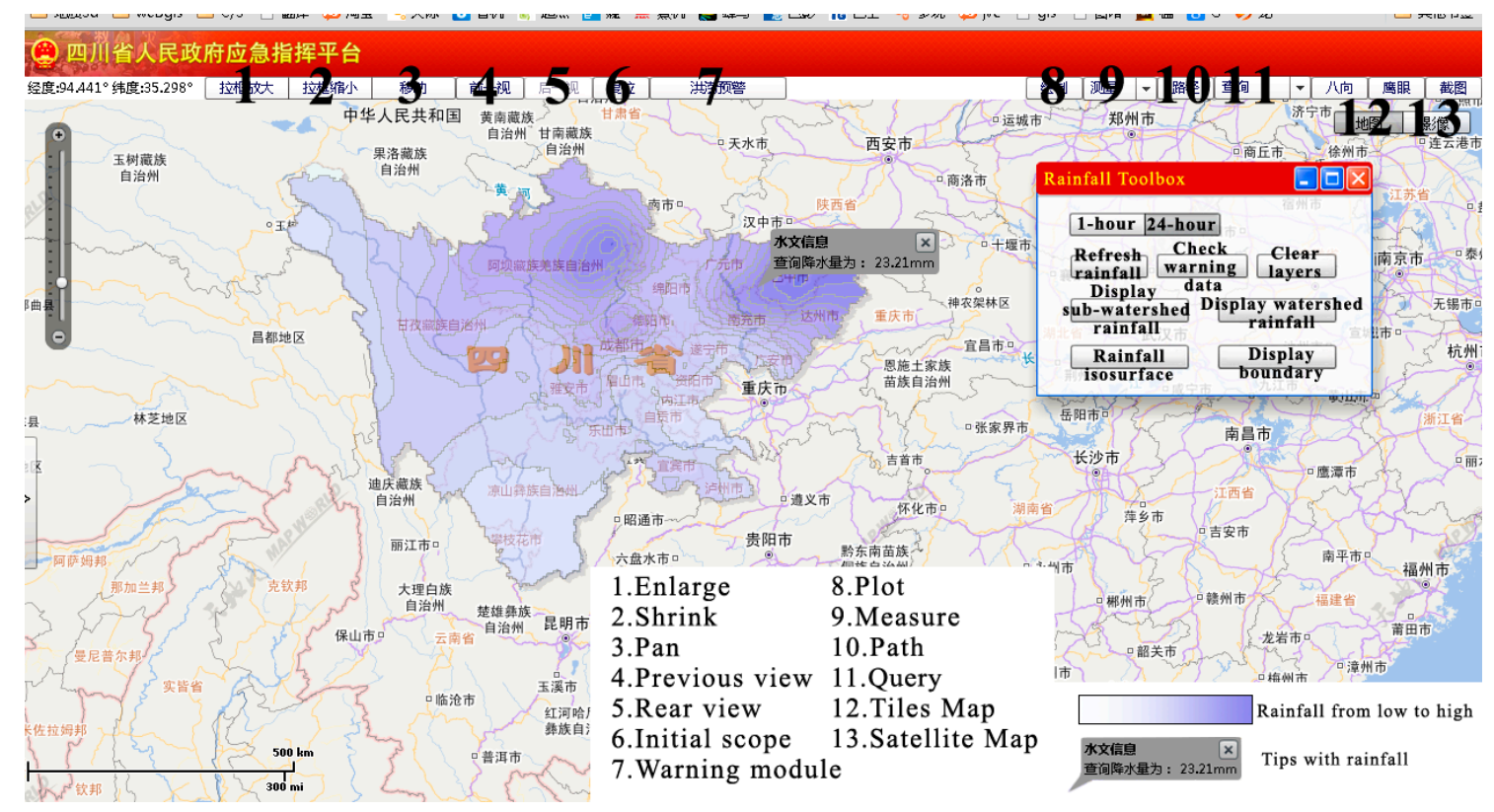

Figure 9. Rainfall display in DSS with browsers interface.

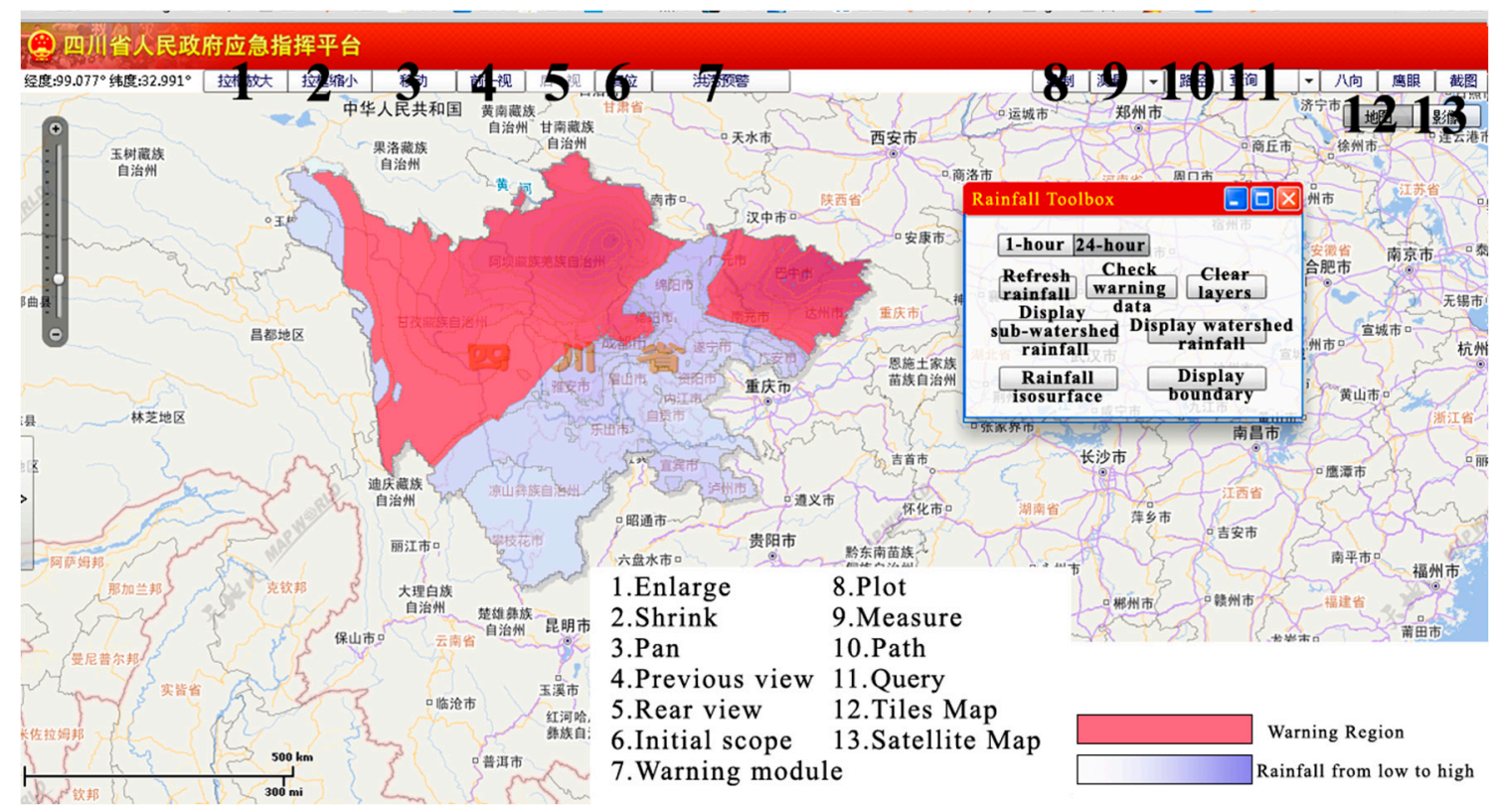

Figure 10. Warn and locate interface.

\section{Other Functions of DSS}

Likewise, DSS can realize complex functions with the powerful geographic processing ability of the ArcGIS Server by releasing different geoprocessing services to free a client from heavy computing tasks and allow it to focus on data presentation. After determining the rainfall alarm location, users can invoke the economic or personnel situation of affected areas with the loss-statistics module, or 
invoke the plotting module annotation arrow and landmarks, or use the path analysis function to find the best travel routes.

The statistics module (Figure 10) is a simulation case for the warning-judgment module. After determining an appropriate buffer range and query topic, users send parameters by JSON to the ArcGIS Server and it executes; then, users can query the population and economic data of the selected region or selected administrative region through the geoprocessing services, which feed the results back in a chart or multiwindow (Figure 11).

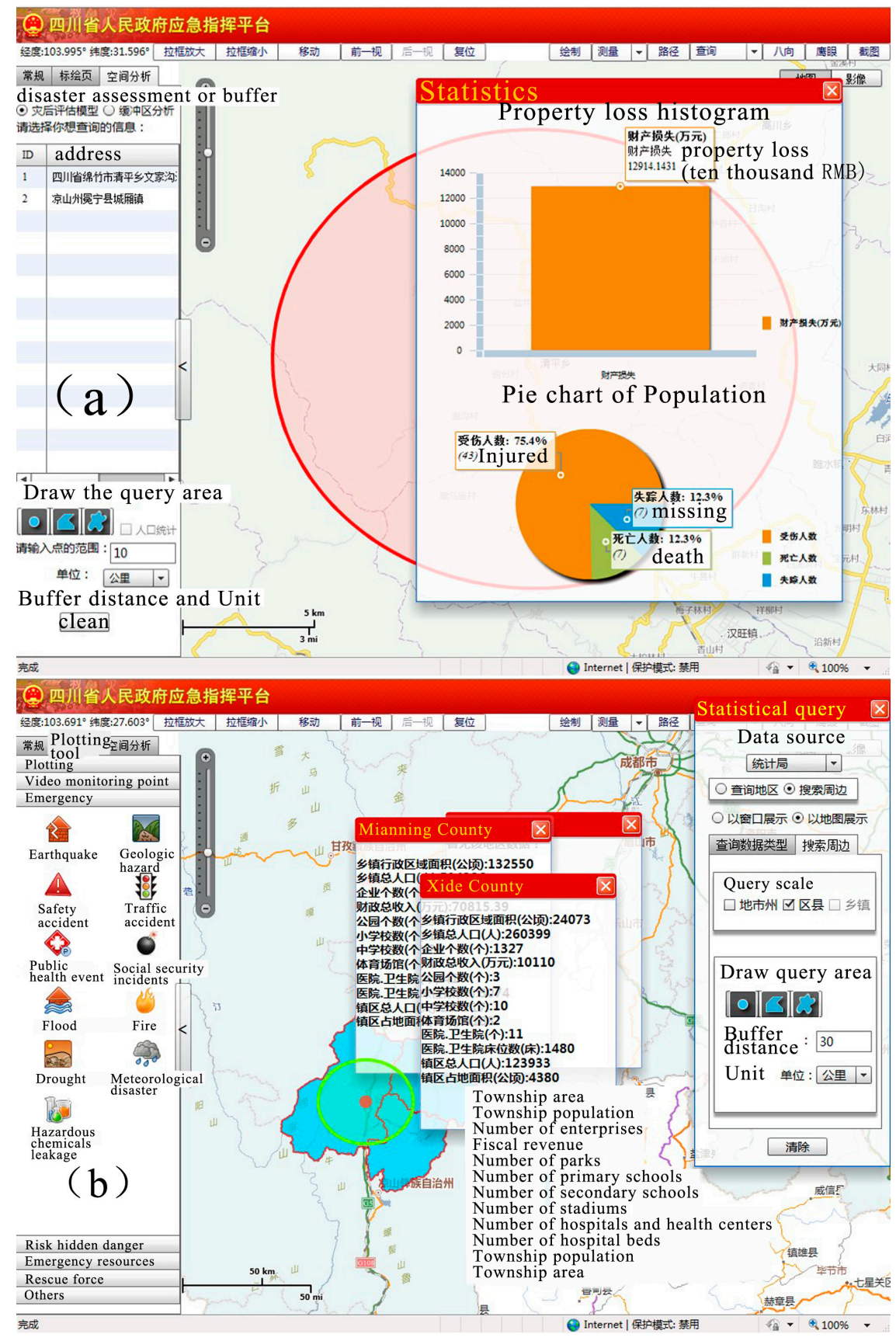

Figure 11. Statistical module: (a) with buffer tools and chart interface; (b) with administrative region and in multiwindow interface.

The map plot module (Figure 12) allows users to make annotations on the map, including marking points, lines, and polygons, and labeling with customized fill colors, contour colors, line styles, and 
some other options. It can be used to mark the allocation of resources and for command and dispatch mapping and situation mapping. The plot module audits user permission. All levels of users can view and modify their own uploaded plotting, though low-level users cannot modify but can only view maps that high-level users have uploaded. All mapping data is stored as XML in the server by Webservice.

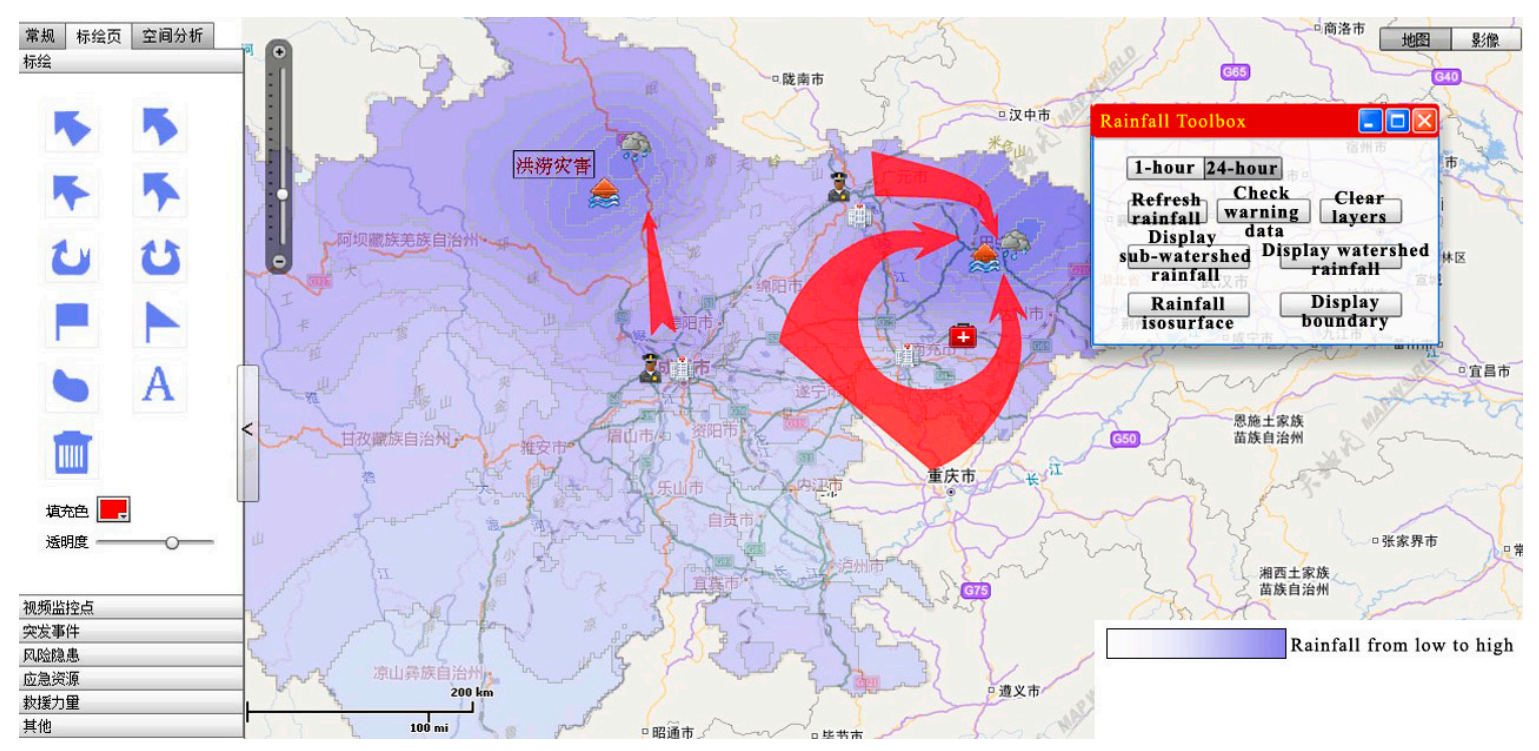

Figure 12. Rainfall display with arrow, pin, and label interface.

\section{Conclusions and Discussion}

Based on geoprocessing services support, a rainfall warning module has been designed within the framework of DSS. The module encapsulates different functions as geoprocessing services, which are deployed on the server with some essential constant data to reduce the network cost of passing parameters. Users can apply the capacities of water conservancy construction against disasters by using historical maximums to avoid the difficulty of determining the threshold value of disaster in DSS. DSS quickly determines the possible regions of flood disaster if the actual data in 1 or $24 \mathrm{~h}$ is greater than the historical average value during the same time period. In addition, DSS provides loss statistics, path analysis, map mapping, public resource query, and other modules. Most of the functional structures of the decision support system are similar to the rainfall warning module, and the computing side is placed in the GIS server to ensure the fluency of the foreground and the friendliness of the interface. In order to ensure the stability and speediness of the system, DSS streamlines the forecast time and precision; hence, it also needs improving. With further development of computer technology and enhancement of the computing ability of hardware, real-time and accurate disaster forecast models will be created in the near future [24], and DSS with the strengths of this model will be constantly improved. This will be one of the major research directions of our team in the future.

Acknowledgments: National Key Technologies Research and Development Program (2016YFB0502605, 2016YFC0803100).

Author Contributions: Linfu Xue, Xiaopeng Leng and Xuejia Sang conceived and designed the experiments; Liang Zhan contributed materials and analysis tools; Xuejia Sang and Jiwen Liu wrote the paper.

Conflicts of Interest: The authors declare no conflict of interest.

\section{References}

1. Wu, Z.Y. Research of Quantitative Rainfall and Real Time Flood Forecasting; Hohai University: Nanjing, China, 2007. 
2. Engel, B.A.; Choi, J.Y.; Harbor, J.; Pandey, S. Web-based DSS for hydrologic impact evaluation of small watershed land use changes. Comput. Electron. Agric. 2003, 39, 241-249. [CrossRef]

3. Salah, H.A.; Mocanu, M.; Florea, A. Development of a prototype ArcGIS-web-based decision application WATERDSS: For water pollution management. In Proceedings of the 7th IEEE International Conference on Electronics, Computers and Artificial Intelligence, Bucharest, Romania, 25-27 June 2015.

4. Kulkarni, A.T.; Mohanty, J.; Eldho, T.I.; Rao, E.P.; Mohan, B.K. A web GIS based integrated flood assessment modeling tool for coastal urban watersheds. Comput. Geosci. 2014, 64, 7-14. [CrossRef]

5. Chen, N.; Hu, C.; Chen, Y.; Wang, C.; Gong, J. Using SensorML to construct a geoprocessing e-Science workflow model under a sensor web environment. Comput. Geosci. 2012, 47, 119-129. [CrossRef]

6. Zhang, W.; Zhou, J.; Liu, Y.; Chen, X.; Wang, C. Emergency evacuation planning against dike-break flood: A GIS-based DSS for flood detention basin of Jingjiang in central China. Nat. Hazards 2016, 81, 1-19. [CrossRef]

7. Wu, H.; Tang, W.; Luo, B.; Lv, Z. Weather services products generation system based on GIS geoprocessing. Comput. Geosci. 2013, 51, 16-21. [CrossRef]

8. Gils, H.C.; Cofala, J.; Wagner, F.; Schöpp, W. GIS-based assessment of the district heating potential in the USA. Energy 2013, 58, 318-329. [CrossRef]

9. Jaroensutasinee, K.; Pheera, W.; Jaroensutasinee, M. Online weather data analysis and visualization tools for applications in ecoinformatics. Earth Sci. Inform. 2014, 7, 205-213. [CrossRef]

10. Zhao, P.; Foerster, T.; Yue, P. The geoprocessing web. Comput. Geosci. 2012, 47, 3-12. [CrossRef]

11. Karami, M.; Rangzan, K.; Saberi, A. Using GIS servers and interactive maps in spectral data sharing and administration: Case study of Ahvaz Spectral Geodatabase Platform (ASGP). Comput. Geosci. 2013, 60, $23-33$. [CrossRef]

12. Li, H.; Zhu, Q.; Yang, X.; Xu, L. Geo-information processing service composition for concurrent tasks: A QoS-aware game theory approach. Comput. Geosci. 2012, 47, 46-59. [CrossRef]

13. Li, X.; Di, L.; Han, W.; Zhao, P.; Dadi, U. Sharing geoscience algorithms in a Web service-oriented environment (GRASS GIS example). Comput. Geosci. 2010, 36, 1060-1068. [CrossRef]

14. Liu, Z.; Guo, S.; Zhang, H.; Liu, D.; Yang, G. Comparative Study of Three Updating Procedures for Real-Time Flood Forecasting. Water Resour. Manag. 2016, 30, 2111-2116. [CrossRef]

15. Qi, H.; Altinakar, M.S. A GIS-based decision support system for integrated flood management under uncertainty with two dimensional numerical simulations. Environ. Model. Softw. 2011, 26, 817-821. [CrossRef]

16. Sandric, I.; Ursaru, P.; Chitu, D.; Mihai, B.; Savulescu, I. Web processing service for landslide hazard assessment. In Proceedings of the EGU General Assembly, Vienna, Austria, 22-27 April 2012; p. 12938.

17. Schröder, D.; Omran, A.F.; Bastidas, M. Automated Geoprocessing Workflow for Watershed Delineation and Classification for Flash Flood Assessment. Int. J. Geoinform. 2015, 11, 31-38.

18. Sun, Z.; Yue, P. The Use of Web 2.0 and Geoprocessing Services to Support Geoscientific Workflows. In Proceedings of the IEEE International Conference on Geoinformatics, Beijing, China, 18-20 June 2010; pp. 1-5.

19. Sun, Z.; Yue, P.; Di, L. GeoPWTManager: A task-oriented web geoprocessing system. Comput. Geosci. 2012, 47, 34-45. [CrossRef]

20. Yang, C.; Chen, N.; Di, L. RESTFul based heterogeneous Geoprocessing workflow interoperation for Sensor Web Service. Comput. Geosci. 2012, 47, 102-110. [CrossRef]

21. Tobler, W.R.A. A Computer Movie Simulating Urban Growth in the Detroit Region. Econ. Geogr. 1970, 46, 234-240. [CrossRef]

22. Zhang, Z.; Ke, C. Monitoring and analysis of changes in a wetland landscape in Xingzi county. Earth Sci. Inform. 2016, 9, 35-45. [CrossRef]

23. Fortuna, L.; Nunnari, G.; Nunnari, S. A new fine-grained classification strategy for solar daily radiation patterns. Pattern Recognit. Lett. 2016, 81, 110-117. [CrossRef]

24. Jones, D.; Jones, N.; Greer, J.; Nelson, J. A cloud-based MODFLOW service for aquifer management decision support. Comput. Geosci. 2015, 78, 81-87. [CrossRef]

(C) 2017 by the authors. Licensee MDPI, Basel, Switzerland. This article is an open access article distributed under the terms and conditions of the Creative Commons Attribution (CC BY) license (http://creativecommons.org/licenses/by/4.0/). 